\title{
Von gebratenem Huhn und faktischer Rechtsweggarantie
}

\author{
integrationXchange - ein transatlantisches Austauschprogramm für Migrationspraktikerlnnen
}

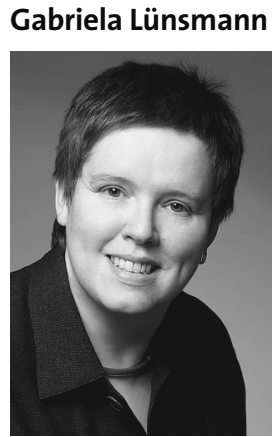

Mitglied der Kommission Öffentliches Recht, Europaund Völkerrecht, zuständig für Migrationsrecht; Rechtsanwältin, Hamburg
Hähnchenteile stapeln sich in der Küche neben einer riesigen Pfanne; im Nebenzimmer werden mehrere quengelnde Kinder von einer Studentin betreut; es ist Mittwochabend kurz vor 19 Uhr und auf der anderen Seite des Flurs diskutieren fünfzig Menschen engagiert auf Spanisch die aktuellen Probleme der Gesundheitsversorgung in ihrem Stadtteil. Der Rechtsanwalt Theo Oshiro erläutert die Ziele einer geplanten Pressekonferenz und nach einigem Hin und Her stehen die TeilnehmerInnen aus der Gruppe für einen Gesprächstermin mit dem Büro des Bürgermeisters fest. Das Geschehen wird interessiert beobachtet von einer Berliner Politologin und einer Hamburger Rechtsanwältin. Wir sind bei „Make the Road by Walking“, einer sog. Community Organisation im New Yorker Stadtteil Brooklyn, die es sich zur Aufgabe gemacht hat, in der überwiegend lateinamerikanischen Gemeinde die Selbstorganisation und Interessenvertretung von Migrantinnen zu fördern und zu unterstützen. Die 1997 gegründete Organisation beschäftigt mittlerweile zahlreiche AnwältInnen und SozialarbeiterInnen, deren Aufgabe es ist, die Menschen in der Gemeinde bei der Selbstorganisation und bei der Durchsetzung ihrer Rechte zu unterstützen. Die Organisation hat Büros in verschiedenen unterprivilegierten Stadtteilen New Yorks; sie verfügt inzwischen über einen Jahresetat von mehreren Millionen Dollar und ihre Stimme wird von den Regierenden in New York gehört.

\section{Best Practice kennenlernen}

Das Kennenlernen von solchen erfolgreichen Projekten der Partizipation und Integration von MigrantInnen ist Ziel des transatlantischen Austauschprogramms integrationXchange, das die Hamburger Körber-Stiftung zusammen mit dem amerikanischen Zweig der Carl Duisberg Gesellschaft durchführt. Das Programm ermöglichte 2007 und 2008 je 15 amerikanischen und deutschen JuristInnen, PolitologInnen, StadtplanerInnen sowie Sozial- und BildungarbeiterInnen für jeweils eine Woche in Hamburg und New York zusammenzukommen, um Arbeitsansätze, Erfahrungen und die jeweilige Best Practice ihrer Herkunftsländer kennenzulernen, zu diskutieren und im Austausch weiterzuentwickeln. Im Anschluss an den einwöchigen Workshop erhielten einzelne deutsche Teilneh-
merInnen die Möglichkeit, im Rahmen eines Extended Stays im Mai 2008 weitere New Yorker Organisationen und Projekte aus ihrem Arbeitsbereich zu besuchen und sich so einen umfassenden Eindruck der New Yorker Infrastruktur zu verschaffen. Im Bereich des Migrationsrechts ermöglichte das ergänzende Programm im Rahmen eines vorher individuell gestalteten Planes den Besuch von zahlreichen Anwalts- und JuristInnenorganisationen, deren VertreterInnen sich regelmäßig viel Zeit nahmen, um ihre Organisationen vorzustellen, Fachgespräche mit verschiedenen JuristInnen zu ermöglichen und bei Bedarf auch weitere Kontakte zu vermitteln.

Unter dem Leitgedanken der Gewährleistung des gleichberechtigten Zugangs von MigrantInnen zu rechtlicher Beratung und anwaltlicher Vertretung im gerichtlichen Verfahren, also einer nicht nur theoretischen, sondern faktischen Rechtsweggarantie, findet sich speziell in New York eine Infrastruktur, die sich maßgeblich von der in Deutschland unterscheidet. Die rechtliche Beratung und anwaltliche Vertretung von MigrantInnen und MigrantInnenorganisationen erfolgt in New York fast ausschließlich durch Non-Profit-Organisationen, die sich sämtlich durch eine sehr hohe Professionalität und eine große Spezialisierung in den verschiedenen Fachgebieten innerhalb des Migrationsrechts auszeichnen.

\section{Das Pro-Bono-Prinzip als Beitrag zur Zivilgesellschaft}

Der gravierendste Unterschied liegt dabei in den Ressourcen, die den Organisationen durch Stiftungsgelder und Pro-Bono-Leistungen großer Anwaltsfirmen zu Verfügung stehen. Ausschlaggebend für diese Spendenbereitschaft ist, dass in den USA als Bedrohung der Zivilgesellschaft bewertet wird, dass faktisch aufgrund der ökonomischen Entwicklung nur noch ein sehr privilegierter Teil der Gesellschaft Zugang zu rechtlicher Vertretung hat.

Es gilt daher für renommierte Anwaltsfirmen durchaus als Aushängeschild, sich mit erheblichen Zuwendungen durch Spendengelder etwa in Form der Finanzierung von Vollzeitstellen in einer NonProfit-Organisation oder durch oder Pro-Bono-Arbeit zu engagieren. Dies hat die Beschäftigung einer Vielzahl von hauptamtlichen JuristInnen mit hoher 
Fachkompetenz in vielen Organisationen zur Folge. Diese fachliche Qualifikation erlaubt den Organisationen auch eine rechtspolitische Positionierung und eine Einflussnahme durch die strategische Auswahl von Präzedenzfällen, die mit hoher Fachkompetenz durch die Instanzen bis zu den Obergerichten vertreten werden können.

Zur nachhaltigen Sicherung dieser Art der Finanzierung ist darüber hinaus bei allen Organisationen die Beschäftigung hauptamtlicher FundraiserInnen selbstverständlich; ihre Aufgabe ist die Akquise von Fördergeldern und Pro-Bono-Zuwendungen und die intensive Zusammenarbeit mit den GeldgeberInnen. Zusätzlich beschäftigen die meisten Organisationen SozialarbeiterInnen und sog. Community Organizer, die den Kontakt zu den MigrantInnenorganisationen in den Stadtteilen aufbauen und pflegen.
Diese Verbindung von hoher juristischer Kompetenz mit den Anliegen der MigrantInnenorganisationen vor Ort in den Gemeinden ist es, die Organisationen wie „Make the Road by Walking“ zu ihrem politischen und gesellschaftlichen Erfolg verhilft. Durch ihre politische Wirksamkeit gelingt es der Organisation, die Menschen in ihrem Stadtteil zur Partizipation in der Einwanderungsgesellschaft zu bewegen. Hierzu gehört auch, dass praktischen Problemen Rechnung getragen wird und während der Sitzungen Kinderbetreuung organisiert und Verpflegung angeboten wird: als das Treffen gegen 22 Uhr endet, zieht denn auch der Duft von gebratenem Huhn durch die Räume und auch die deutschen Besucherinnen werden noch mit einem warmen Abendessen versorgt, bevor sie sich mit ganz neuen Eindrücken auf den Heimweg machen.

\section{Die OLG-Präsidentin}

\section{Gedenkschrift für Henriette Heinbostel}

Herausgegeben von Konstanze Görres-Ohde, Monika Nöhre und Anne-José Paulsen

Berliner Wissenschaftsverlag (BWV), Berlin 2007

ISBN 978-3-8305-1444-2

Kartoniert, 177 Seiten, 29,00 EUR

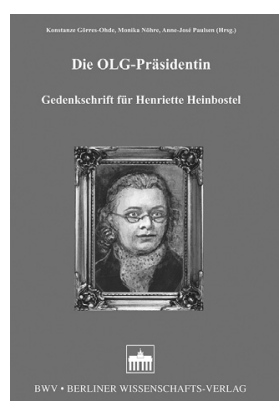

Henriette Heinbostel war aus rechtsund frauenpolitischer Sicht eine bedeutende Person. Vor allem anderen war sie weit und breit die erste OLG-Präsidentin. Den drei Herausgeberinnen ist für die vorliegende Gedenkschrift sehr zu danken - ist sie doch, wie Bundesjustizministerin Brigitte Zypries in ihrem Vorwort betont, eine der sehr sehr wenigen Fest- und Gedenkschriften, die Juristinnen gewidmet sind. Die folgenden Beiträge namhafter Richterinnen, Rechtsanwältinnen, Professorinnen, Senatorinnen und einer Journalistin über Henriette Heinbostel im Besonderen und die Karrieren von Juristinnen in der Bundesrepublik im Allgemeinen bringen alles das zur Sprache, womit sich Juristinnen in den vergangenen Jahrzehnten abplagten und es teilweise immer noch tun.

Die Vereinbarkeit von Familie und Beruf wird heute wie damals als ein Problem der Frauen diskutiert, obwohl die Kinder üblicherweise zwei Elternteile haben. Ein Fortschritt liegt hier sicher schon darin, dass die Ehemänner seit 1958 ihren Frauen die erst durch das Gleichberechtigungsgesetz aus der Welt geschaffte Arbeitserlaubnis nicht mehr verweigern können. Der 50ste Jahrestag des Gleichberechtigungsgesetzes wird derzeit groß gefeiert. Doch die Frage, ob Frauen eher durch das Abfassen von Urteilen und Leiten eines Gerichtes Zufriedenheit gewinnen oder allein durch das Backen von Apfelkuchen für die Familie, scheint heute wieder ernsthaft diskutiert zu werden. Auch die Frage missratener, weil fremdbetreuter Kinder berufs- tätiger Mütter - Henriette Heinbostels 17-jährige Tochter Renate amüsierte sich bei der Amtseinführung ihrer Mutter mit dem Wachtmeister im dunklen Keller des OLG - ist immer noch nicht vom Tisch.

Damals wie heute hatten offenbar die Frauen die besseren Examensergebnisse. Heinriette Heinbostel etwa schloss Studium und Referendariat mit Auszeichnung ab, während der biologische Vater ihrer Tochter Renate, Friedrich Gottlob Nagelmann, lediglich ein „besonders befriedigend“ erreichte. Doch in den höheren Positionen spiegeln sich die Examensergebnisse in der Regel auch heute nicht wider. OLG-Präsidentinnen wie Henriette Heinbostel sind Mangelware. Vier der 24 Oberlandesgerichte werden derzeit von Frauen geleitet: von Anne-José Paulsen (Düsseldorf, seit März 2002), Monika Nöhre (Berlin, seit August 2002), Erika Andreß (Hamburg, seit Juli 2007) und Uta Fölster (Schleswig, seit Januar 2008). Üppig ist die Zahl ihrer Vorgängerinnen nicht; zu ihnen zählen außer Henriette Heinbostel Helga Oltrogge (OLG Celle, 1989-2006), Gisela Knobloch (KG Berlin, 1990-2001), Hildegund Holzheid (OLG München, 1992-2001), Gertrud Neuwirth (OLG Naumburg, 1996-2003), Brigitte Tilmann (OLG Frankfurt, 1998-2006), Edda Huther (OLG München, 2001-2005) und Konstanze Görres-Ohde (OLG Schleswig, 2002-2007).

Die Lektüre der Gedenkschrift bietet den Leserinnen einige Déjà vu-Erlebnisse. Was ziehe ich bloß an, ist eine der Fragen, die auch erfolgreiche Juristinnen und solche, die es werden wollen, sich immer wieder und wieder stellen, während die Juristen jahraus jahrein zum bewährten schwarzen Anzug greifen. Henriette Heinbostels Ratschlag an Prof. Dr. Ninon Colneric anlässlich ihrer Kandidatur als LAG-Präsidentin 1989 kann da schon hilfreich sein: „Ziehen Sie sich ein bisschen anders an. Natürlich nicht so verspielt, dass man Sie in dieselbe Kategorie steckt wie Sekretärinnen, die Richter verführen wollen. Aber kopieren Sie auch nicht die Männer. Sie müssen bei aller Sachlichkeit und Nüchternheit einen weiblichen Akzent setzen sonst kriegen die Jungs nämlich Angst.“

Das höchst lesenswerte Buch hält weitere interessante Einund Aussichten parat.

(Anke Gimbal) 